\title{
A study on the biological function of heat shock factor 1 proteins in breast cancer
}

\author{
XIAOLAN WANG, DIANLONG ZHANG, MINGQIAN CAO, JINLING BA, BIN WU, TAO LIU and CHEN NIE \\ Department of Breast and Thyroid Surgery, Affiliated Zhongshan Hospital of Dalian University, \\ Dalian, Liaoning 116000, P.R. China
}

Received September 5, 2017; Accepted June 15, 2018

DOI: $10.3892 / \mathrm{ol} .2018 .9062$

\begin{abstract}
The present study aimed to investigate the effect of HSF1 proteins on cell proliferation, apoptosis and invasion of breast cancer. The Michigan Cancer Foundation-7 (MCF-7) HSF1-knocked down stable cell line (experimental group) and control cell line (control group) were obtained using a lentivirus assay, and the effects of HSF1 knockdown on the proliferation, tumor formation, apoptosis and invasion ability were analyzed, respectively. The effects of HSF1 on downstream signals were analyzed using western blotting. Western blotting results showed that lentivirus successfully established a HSF1 knockdown stable cell line of MCF-7. Compared with the control group, the growth rate of MCF-7 cells in the experimental group was significantly decreased $(\mathrm{P}<0.05)$. Flow cytometry showed that the proportion of apoptosis in the control group was significantly lower than that of the experimental group $(\mathrm{P}<0.05)$. Notably, the invasion ability of cells in the control group was significantly higher than that in the experimental group $(\mathrm{P}<0.05)$. Compared with cells in the control group, the levels of heat shock protein (HSP)70, HSP90, anti-apoptotic protein B-cell lymphoma 2 (Bcl-2) and macrophage migration inhibitory factor (MIF) in the experimental group were significantly downregulated, whereas the level of Bax was significantly increased $(\mathrm{P}<0.05)$. In conclusion, HSF1 protein, as a transcription factor, regulates the expression levels of HSP70, HSP90, MIF, Bcl-2 and Bax, thus controlling the proliferation, apoptosis and invasion of cells. These findings suggest HSF1 protein as a potential target for the treatment of breast cancer.
\end{abstract}

Correspondence to: Dr Dianlong Zhang, Department of Breast and Thyroid Surgery, Affiliated Zhongshan Hospital of Dalian University, 6 Jiefang Street, Zhongshan, Dalian, Liaoning 116000, P.R. China

E-mail: zd104111970@163.com

Key words: heat shock factor 1, short hairpin RNA, heat shock protein 70 , heat shock protein 90 , macrophage migration inhibitory factor

\section{Introduction}

Breast cancer is one of the most common types of gynecological malignant tumors, which has become one of the major disease types that seriously affect female's physical and psychological health (1). The pathogenesis of breast cancer has long been the focus of clinical and basic studies. With the continuous development of molecular biology, more and more genes related to the incidence of breast cancer have been found. Heat shock factor 1 (HSF1) is a transcription factor for heat shock proteins (HSPs). It can specifically bind to heat shock original proteins that are necessary for the upstream transcription of the HSP gene promoter, thus activating the transcription of HSPs as well as unknown proteins $(2,3)$. Current studies have shown that breast cancer, lung cancer and other malignant tumors, and it is closed related to clinicopathologic features and clinical prognosis (4,5). HSF1 is highly expressed in esophagus cancer, which can be used as a marker for clinical diagnosis of oral cancer (6). Another study showed that the expression of HSF1 was significantly increased in esophageal carcinoma tissues and correlated with the prognosis of patients (7). Studies on cell level have shown that HSF1 plays an important role in tumorigenesis, apoptosis and proliferation $(8,9)$. Reducing HSF1 can significantly inhibit the growth of tumor cells and promote apoptosis of tumor cells (10). However, what role it plays in tumors is still unclear. In this study, the effects of HSF1 on biological functions of breast cancer cells were analyzed, and the possible molecular mechanism was explored. HSF1 is expected to provide certain targets for the treatment of breast cancer.

\section{Materials and methods}

Experimental materials. Balb/c nude mice, weighing 18-20 g were purchased from Shanghai SLAC Laboratory Animal Co., Ltd.; Michigan Cancer Foundation-7 (MCF-7) breast cancer cell lines were purchased from American Type Culture Collection (ATCC); MCF-7 cells are estrogen dependent breast cancer cells. Embedding estrogen can promote the formation of breast cancer solid tumor.Dulbecco's modified Eagle's medium(DMEM), fetal bovine serum (FBS), trypsin and streptomycin were purchased from Gibco; Thermo Fisher Scientific, Inc., (Waltham, MA, USA); 3-(4,5-Dimethylthiazol-2-yl)-2,5-diphenyltetrazolium bromide (MTT) was purchased from Sigma-Aldrich; Merck 
KGaA, (Darmstadt, Germany); 24 mm Transwell ${ }^{\circledR}$ with $3 \mu \mathrm{m}$ Pore was purchased from Corning Incorporated, Corning, NY, USA (cat. no. 3452); HSF1 lentiviruses and no-load control viruses were packaged by Shandong Vigene Biosciences Co., Ltd. (Shandong, China); Annexin V-fluorescein isothiocyanate (FITC) Apoptosis Detection kit was purchased from Beyotime Institute of Biotechnology, (Haimen, China); 70 kilodalton HSP (HSP70), HSP90, B-cell lymphoma 2 (Bcl-2), Bcl-2-associated $\mathrm{X}$ (Bax) and macrophage migration inhibitory factor (MIF) monoclonal antibodies were purchased from Santa Cruz Biotechnology, Inc., (Dallas, TX, USA). Other reagents were analyzed and purified by companies in China. The present study was approved by the Ethics Committee of Affiliated Zhongshan Hospital of Dalian University (Dalian, China).

Construction and verification of stable HSF1-knockdown cell lines. MCF-7 cells cultured to the logarithmic growth phase were inoculated into 6-well plates. After the cells were adherent to the wall, $100 \mu \mathrm{l}$ HSF1 short hairpin ribonucleic acid (shRNA) viruses and control viruses were added to the medium in two wells. Cells were further cultured for $24 \mathrm{~h}$, and then the medium was replaced. At $48 \mathrm{~h}$ after infection, the infection of viruses was observed under a fluorescence microscope (IX70; Olympus, Tokyo, Japan), and puromycin was added to cells for killing the uninfected cells. The survived cells were successfully infected cells. Some cells were collected, and the expression level of HSF1 proteins was analyzed by western blotting.

Analysis of the effect of HSF1 on the proliferation of MCF-7 cells by MTT. The cells in the control and experimental groups were cultured to the logarithmic growth phase. After the digestion with trypsin, the cell density was adjusted to $1 \times 10^{5} / \mathrm{ml}$, and $100 \mu \mathrm{l}$ cells were inoculated in 48 -well plates. After the cells were adherent to the wall, $20 \mu 1(5 \mathrm{mg} / \mathrm{ml})$ MTT was added to cells at 12,24, 36 and $48 \mathrm{~h}$ after incubation, respectively. After that, cells were further cultured in an incubator for $3 \mathrm{~h}$. Then the medium was removed, and cells in the two groups were cultured for $6 \mathrm{~h}$, respectively. After that, the medium was discarded, and each well was added with $100 \mu$ l dimethyl sulfoxide (DMSO) and shaken evenly. The optical density (OD) value of cells in each well at the wavelength of $570 \mathrm{~nm}$ was detected using a microplate reader (Bio-Rad, Hercules, CA, USA), the survival rate of cells was calculated, and the corresponding concentration-survival curve was drawn.

Analysis of the effect of HSF1 on the apoptosis of MCF-7 cells by flow cytometry. Cells in the control and experimental groups were digested with trypsin and centrifuged, followed by the washing with phosphate-buffered saline (PBS) after centrifugation at $1,000 \mathrm{x} \mathrm{g}$ for $5 \mathrm{~min}$. Then the cells were gently resuspended with PBS and counted. A total of 5-10x10 were taken to be centrifuged at $1,000 \mathrm{xg}$ for $5 \mathrm{~min}$, and after the supernatant was discarded, $195 \mu \mathrm{l}$ Annexin V-FITC solution was added to gently resuspend the cells. A total of $5 \mu \mathrm{l}$ Annexin V-FITC and $10 \mu \mathrm{l}$ propyl iodide were added and gently mixed with the solution. Afterwards, the cells were incubated at room temperature $\left(20-25^{\circ} \mathrm{C}\right)$ for $10-20 \mathrm{~min}$ in a dark place and placed in an ice bath, and then they were analyzed by flow cytometry (FACSCalibur; BDBiosciences, Detroit, MI, USA). Annexin V-FITC showed green fluorescence, and propidium iodide (PI) showed red fluorescence (11-13).

Analysis of the effects of HSF1 on the invasion of MCF-1 cells by Transwell assay. The chamber was placed in culture plates. A total of $300 \mu \mathrm{l}$ pre-warmed serum-free medium was added to the upper chamber and placed for standing at room temperature for 15-30 min so as to rehydrate the matrix gel. Then the remaining culture medium was extracted. After cells in the control and experimental groups grew to the logarithmic growth phase, they were digested with trypsin. At the end of digestion, the culture medium was discarded by centrifugation at $1,500 \mathrm{x} \mathrm{g}$ for $5 \mathrm{~min}$ at $4^{\circ} \mathrm{C}$, and the cells were washed twice with PBS and resuspended in the serum-free medium containing bovine serum albumin (BSA). The cell density was adjusted to $1 \times 10^{5} /$ well. A total of $100 \mu 1$ cell suspension was taken and placed into the Transwell chamber. Generally, $500 \mu 1$ medium containing FBS was added into the lower chamber, and the routine culture was conducted for $48 \mathrm{~h}$. The cells were then stained with crystal violet for calculating the number of invasive cells (11-13).

Analysis and verification of proteomics of cells both in the control and experimental groups by mass spectrometry. The cells in the control and experimental groups were cultured to the logarithmic growth phase. After the digestion with trypsin, the cells were fully lysed with the cell lysate and centrifuged at $10,500 \mathrm{x}$ g for $20 \mathrm{~min}$. The total protein was quantitatively analyzed. The content of the total protein in the two groups was adjusted to $1 \mathrm{mg} / \mathrm{ml}$, and then proteomics analysis was conducted to explore the difference in the expression of cell proteins between the two groups. The results of mass spectrometry were then analyzed by western blotting. Operations: Cell lysates in the two groups were treated with sodium dodecyl sulfate polyacrylamide gel electrophoresis (SDS-PAGE), after which target proteins were transferred to the nitrocellulose (NC) membrane and blocked with 5\% skimmed milk powder for $1 \mathrm{~h}$. After that, target proteins were diluted with rabbit monoclonal HSF1 antibody (dilution, 1/500; cat. no. ab52757; Abcam, Cambridge, MA, USA) and incubated overnight at $4^{\circ} \mathrm{C}$. After washing with PBS with Tween 20 (PBST) 3 times the next day, HRP-labeled secondary goat anti-rabbit (HRP) IgG antibody (dilution, 1/2,000; cat. no. ab6721, Abcam) was used for incubation at room temperature for $1 \mathrm{~h}$. At the end of that, proteins were washed with PBST 3 times for $5 \mathrm{~min}$, and then enhanced chemiluminescence (ECL) (Merck Millipore, Billerica, MA, USA) was used for color development with $\beta$-tubulin as an internal reference.

Effects of HSF1 on tumorigenesis ability of MCF-7 cells in vivo. The nude mice were embedded with estrogenic sustained release tablets ( $0.36 \mathrm{mg} /$ tablet; Innovative Research, Novi, MI, USA) subcutaneously before inoculation of the tumor. After 3 days of inoculation, $1 \times 10^{7}$ MCF-7 control cells and HSF1 knockout MCF-7 cells were inoculated in the subaxillary fat pad of nude mice respectively. The tumor was observed every day and the volume of the tumor was measured. The longest diameter was $1.5 \mathrm{~cm}$. The volume of the tumor was $=1 / 2$ length $\mathrm{x}$ width ${ }^{2}$. 

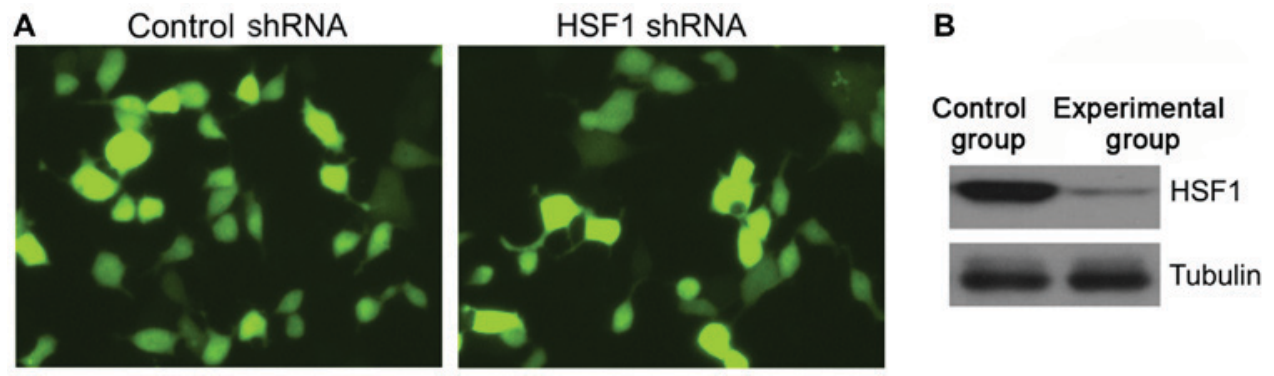

Figure 1. Analysis of infection rate and knockdown rate of lentiviruses. (A) Immunofluorescence was used to analyze the infection efficiency of cells both in the control and observation groups after lentivirus infection (magnification, x400). (B) The expression of HSF1 protein in the cells of the control and observation groups were analyzed by western blotting.
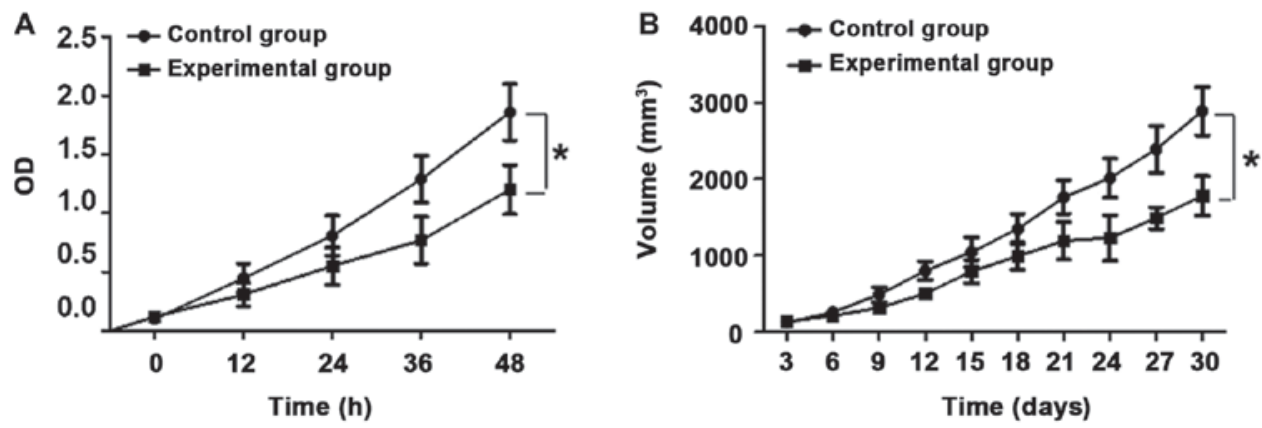

Figure 2. Effects of HSF1 on the proliferation of breast cancer at the cell and animal level. (A) MTT was used to analyze the proliferative ability of cell in the control group and the observation group. (B) The growth rate of the control group and the observation group in vivo was analyzed by tumorigenesis experiment. ${ }^{*} \mathrm{P}<0.05$.

Statistical analysis. All the data were analyzed using Statistical Product and Service Solutions (SPSS, Inc., Chicago, IL, USA) v.13.0 statistical software. Measurement data were expressed as mean \pm standard deviation and compared using the t-test. $\mathrm{P}<0.05$ was considered to indicate a statistically significant difference.

\section{Results}

Construction of stable HSF1-knockdown cell lines. As shown in Fig. 1, lentivirus-mediated shRNA was used to construct stable HSF1-knockdown cell lines in this study. After the puromycin screening for $72 \mathrm{~h}$, green fluorescent proteins (GFPs) could be seen in approximately $85 \%$ of the cells, suggesting that lentivirus-mediated shRNA is successfully infected in MCF-7 cells. Western blotting further verified in this study that the HSF1/Tubulin ratio $(0.24 \pm 0.10)$ of the experimental group was significantly decreased compared with that $(2.13 \pm 0.25)$ of the control group, and the difference was statistically significant $(\mathrm{P}<0.05)$.

Effects of HSF1 on cell proliferation and tumorigenesis ability. As shown in Fig. 2, the cell growth rate of the experimental group significantly became slower compared with that of the control group, and the difference was statistically significant $(\mathrm{P}<0.05)$. In vivo tumor formation assay confirmed that the tumor formation rate and growth rate of cells in the control group were significantly increased compared with those of cells in the experimental group, and the differences were statistically significant $(\mathrm{P}<0.05)$.
The effects of HSF1 on the apoptosis level of cells. As shown in Fig. 3, the proportion of apoptotic cells in the control group was $(2.38 \pm 0.58)$, and that in the experimental group was $(12.35 \pm 2.35)$. The difference was statistically significant $(\mathrm{P}<0.05)$.

The effects of HSF1 on the invasion ability of cells. The invasion ability of cells $(372.29 \pm 53.21) /$ well in the experimental group was significantly decreased compared with that of cells in the control group (143.29 exper)/well, and the difference was statistically significant $(\mathrm{P}<0.05)$.

The effect of HSF1 on the expression of downstream target proteins. In this experiment, the proteomics analysis was conducted for cells both in the control and experimental groups. The results revealed that there were differences in the expression of HSP70, HSP90, anti-apoptotic Bcl-2 proteins, Bax proteins and MIFs. Western blotting further verified in this experiment that the levels of HSP70, HSP90, anti-apoptotic Bcl-2 proteins and MIFs in the experimental group were significantly downregulated compared with those in the control group, and the differences were statistically significant $(\mathrm{P}<0.05$; Fig. 4).

\section{Discussion}

HSF1, as a transcription factor, can promote the transcription and expression of HSP genes. These HPSs, as molecular chaperones, play an important role in maintaining the toxicity of proteins in the body and the body's metabolism, growth and 


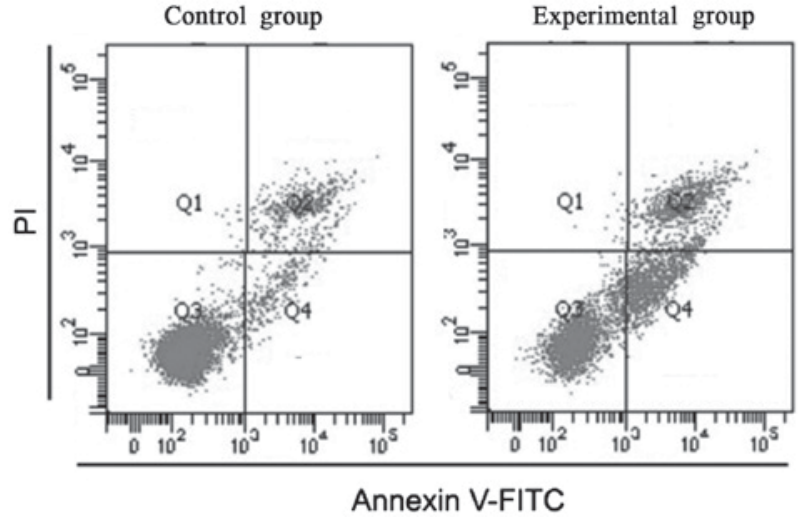

Figure 3. Effects of HSF1 on the apoptosis level of cells. Flow cytometry was used to analyze the changes of the apoptosis level both in the control and observation groups.
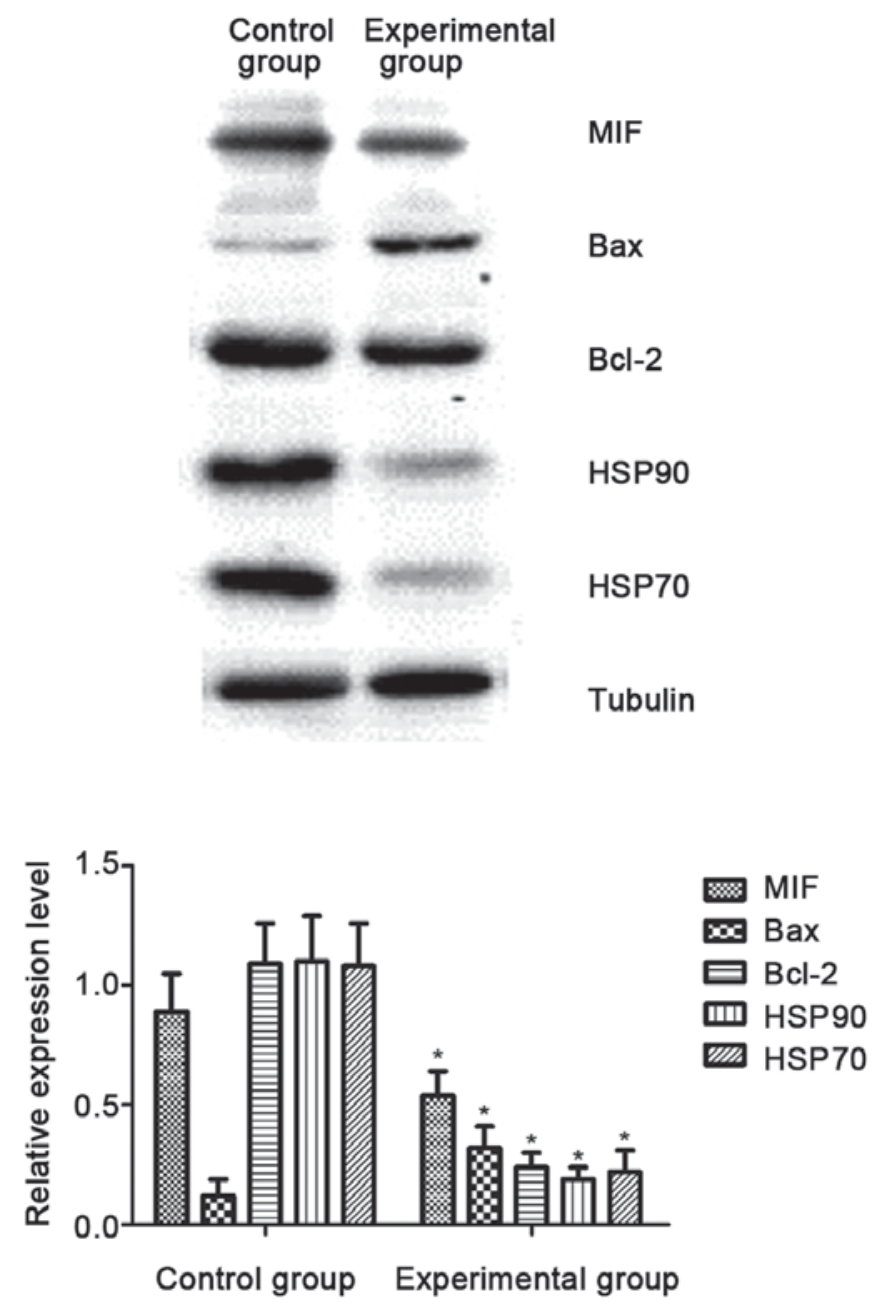

Figure 4. Analysis of the effects of HSF1 knockdown on the expression of downstream proteins by western blotting. The levels of HSP70, HSP90, anti-apoptotic $\mathrm{Bcl}-2$ proteins and MIFs in the experimental group were significantly downregulated compared with those in the control group. The levels of Bax protein in the experimental group were significantly upregulated compared with those in the control group. ${ }^{*} \mathrm{P}<0.05$. MIFs, migration inhibitory factors.

development. However, tumor cells are more dependent on the function of HSF1 than normal cells, as studies have shown that
HSF1 is necessary in regulating tumor cell abnormal signals, inhibiting mitosis so as to increase genomic aneuploidy, inhibiting tumor cell apoptosis and promoting tumor cell metastasis and metabolism. HSF1 can only play its role in the form of trimerization, and then proteins shift into the nucleus and highly phosphorylated, thus binding to heat shock elements (HSEs) and activating the activation of downstream genes $(14,15)$. Although it is highly expressed in many tumor tissues, its effects on the cell biological function and how it affects the cell biological function are not clear.

The present study showed that the proliferation rate and tumor growth rate of HSF1-knockdown cells were significantly decreased, indicating that HSF1 may be involved in the proliferation of breast cancer cells. From the perspective of apoptosis, it was found that the apoptosis rate of HSF1-knockdown MCF-7 breast cancer cells was significantly higher than that of the control cells, indicating that the overexpression of HSF1 significantly reduces cell apoptosis and promotes cell proliferation in another aspect. In addition, the invasion ability of cells reflected the features of the metastasis and infiltration of tumor cells at a particular level. It was also found that the invasion ability of HSF1-knockdown MCF-7 cells was significantly decreased, manifesting that HSF1 is related to the invasion ability of MCF-7 cells.

Recent studies have shown that the AKT-mTOR signaling pathway regulates the activation of HSF1 protein. The activation of HSF1 significantly upregulated the transcription and translation of the oncogene, resulting in abnormal cell proliferation or migration and invasion, and the deterioration of cell properties (16-18). In order to further analyze how HSF1 knockdown affects the above biological behaviors of MCF-7 cells, proteomics techniques were used to analyze the difference in protein expression between control cells and HSF1-knockdown cells. The results revealed that the expression of HSP70, HSP90, anti-apoptotic $\mathrm{Bcl}-2$ proteins, pro-apoptotic Bax proteins and MIFs in the two groups were significantly different. HSP70 and HSP90 are two important HSPs and one of the target proteins of HSF1, which are currently found to be highly expressed in many tumor tissues for promoting tumor development and metastasis $(19,20)$. Anti-apoptotic Bcl-2 proteins and pro-apoptotic Bax proteins are the key proteins determining the fate of cells, and $\mathrm{Bcl}-2 / \mathrm{Bax}$ ratio variation determines the cells' tendency to apoptosis or normality (21). MIF is a unique cytokine that promotes the development of malignant tumors. A previous study has shown that the expression level of MIF is inseparable from tumor progression and tumor angiogenesis (22). This study revealed that the levels of HSP70, HSP90, anti-apoptotic Bcl-2 proteins and MIFs were significantly downregulated, and the level of Bax was significantly increased after HSF1 knockdown, and this result was consistent with the above-mentioned variation trend of cell biological behaviors.

In summary, HSF1 may promote cell proliferation, inhibit cell apoptosis and increase cell invasion so as to promote disease progression by acting on its downstream signaling molecules, and it is expected to be used as a target for the treatment of tumors.

\section{Acknowledgements}

Not applicable. 


\section{Funding}

No funding was received.

\section{Availability of data and materials}

The datasets used and/or analyzed during the present study are available from the corresponding author on reasonable request.

\section{Authors' contributions}

XW, DZ and CN conceived and designed the study. MC, JB, BW and TL collected, analyzed and interpreted the data. XW drafted and revised the manuscript critically for important intellectual content. All authors read and approved the final manuscript.

\section{Ethics approval and consent to participate}

The present study was approved by the Ethics Committee of Affiliated Zhongshan Hospital of Dalian University (Dalian, China).

\section{Patient consent for publication}

Not applicable.

\section{Competing interests}

The authors declare that they have no competing interests.

\section{References}

1. Siegel RL, Miller KD and Jemal A: Cancer statistics, 2017. CA Cancer J Clin 67: 7-30, 2017.

2. Chen YF, Wang SY, Yang YH, Zheng J, Liu T and Wang L: Targeting HSF1 leads to an antitumor effect in human epithelial ovarian cancer. Int J Mol Med 39: 1564-1570, 2017.

3. Zhou Z, Li Y, Jia Q, Wang Z, Wang X, Hu J and Xiao J: Heat shock transcription factor 1 promotes the proliferation, migration and invasion of osteosarcoma cells. Cell Prolif 50, 2017.

4. Wang B, Lee CW, Witt A, Thakkar A and Ince TA: Heat shock factor 1 induces cancer stem cell phenotype in breast cancer cell lines. Breast Cancer Res Treat 153: 57-66, 2015.

5. Cui J, Tian H and Chen G: Upregulation of nuclear heat shock factor 1 contributes to tumor angiogenesis and poor survival in patients with non-small cell lung cancer. Ann Thorac Surg 100: 465-472, 2015

6. Tsukao Y, Yamasaki M, Miyazaki Y, Makino T, Takahashi T, Kurokawa Y, Miyata H, Nakajima K, Takiguchi S, Mimori K, et al: Overexpression of heat-shock factor 1 is associated with a poor prognosis in esophageal squamous cell carcinoma. Oncol Lett 13: 1819-1825, 2017.

7. Kim SA, Kwon SM, Yoon JH and Ahn SG: The antitumor effect of PLK1 and HSF1 double knockdown on human oral carcinoma cells. Int J Oncol 36: 867-872, 2010.
8. Ishiwata J, Kasamatsu A, Sakuma K, Iyoda M, Yamatoji M, Usukura K, Ishige S, Shimizu T, Yamano Y, Ogawara K, et al: State of heat shock factor 1 expression as a putative diagnostic marker for oral squamous cell carcinoma. Int J Oncol 40: 47-52, 2012.

9. Nguyen HA and Kim SA: 2'-Hydroxycinnamaldehyde induces apoptosis through HSF1-mediated BAG3 expression. Int J Oncol 50: 283-289, 2017.

10. Cigliano A, Wang C, Pilo MG, Szydlowska M, Brozzetti S, Latte G, Pes GM, Pascale RM, Seddaiu MA, Vidili G, et al: Inhibition of HSF1 suppresses the growth of hepatocarcinoma cell lines in vitro and AKT-driven hepatocarcinogenesis in mice. Oncotarget 8: 54149-54159, 2017.

11. Dong C, Zhang L, Sun R, Liu J, Yin H, Li X, Zheng X and Zeng H: Role of thioredoxin reductase 1 in dysplastic transformation of human breast epithelial cells triggered by chronic oxidative stress. Sci Rep 6: 36860, 2016.

12. Yan X, Chen X, Liang H, Deng T, Chen W, Zhang S, Liu M, Gao X, Liu Y, Zhao C, et al: miR-143 and miR-145 synergistically regulate ERBB3 to suppress cell proliferation and invasion in breast cancer. Mol Cancer 13: 220, 2014.

13. Tao WY, Wang CY, Sun YH, Su YH, Pang D and Zhang GQ: MicroRNA-34c suppresses breast cancer migration and invasion by targeting GIT1. J Cancer 7: 1653-1662, 2016.

14. Antonietti P, Linder B, Hehlgans S, Mildenberger IC, Burger MC, Fulda S, Steinbach JP, Gessler F, Rödel F, Mittelbronn M and Kögel D: Interference with the HSF1/HSP70/BAG3 pathway primes glioma cells to matrix detachment and $\mathrm{BH} 3$ mimetic-induced apoptosis. Mol Cancer Ther 16: 156-168, 2017.

15. Sawai M, Ishikawa Y, Ota A and Sakurai H: The proto-oncogene JUN is a target of the heat shock transcription factor HSF1. FEBS J 280: 6672-6680, 2013.

16. Chou SD, Prince T, Gong J and Calderwood SK: mTOR is essential for the proteotoxic stress response, HSF1 activation and heat shock protein synthesis. PLoS One 7: e39679, 2012.

17. Nicoletti F, Fagone P, Meroni P, McCubrey J and Bendtzen K: mTOR as a multifunctional therapeutic target in HIV infection. Drug Discov Today 16: 715-721.2011.

18. Sokolosky ML, Stadelman KM, Chappell WH, Abrams SL, Martelli AM, Stivala F, Libra M, Nicoletti F, Drobot LB, Franklin RA, et al: Involvement of Akt-1 and mTOR in sensitivity of breast cancer to targeted therapy. Oncotarget 2: 538-550, 2011.

19. Horibe T, Torisawa A, Kohno M and Kawakami K: Synergetic cytotoxic activity toward breast cancer cells enhanced by the combination of Antp-TPR hybrid peptide targeting Hsp90 and Hsp70-targeted peptide. BMC Cancer 14: 615, 2014.

20. Proia DA and Kaufmann GF: Targeting heat-shock protein 90 (HSP90) as a complementary strategy to immune checkpoint blockade for cancer therapy. Cancer Immunol Res 3: 583-589, 2015.

21. Teymournejad O, Mobarez AM, Hassan ZM and Talebi Bezmin Abadi A: Binding of the Helicobacter pylori OipA causes apoptosis of host cells via modulation of Bax/Bcl-2 levels. Sci Rep 7: 8036, 2017.

22. Nobre CC, de Araújo JM, Fernandes TA, Cobucci RN, Lanza DC, Andrade VS and Fernandes JV: Macrophage migration inhibitory factor (MIF): Biological activities and relation with cancer. Pathol Oncol Res 23: 235-244, 2017.

This work is licensed under a Creative Commons

Attribution-NonCommercial-NoDerivatives 4.0 International (CC BY-NC-ND 4.0) License. 